\title{
An Improved Performance of Switched Reluctance Motor Drives Using Z-Source Inverter with the Control Rule Reduction of Fuzzy Logic Based PI Controller
}

\author{
M.Hari Prabhu \\ M.E-Power Electronics and Drives, Paavai college of Engineering, Anna University-Chennai
}

\begin{abstract}
This paper is based on the improved performance of the closed loop control of Switched Reluctance Motor drives using Z-Source Inverter with the control rule reduction of $5 x 5$ using Fuzzy Logic Controller(FLC) with the output scaling factor(SF)self-tuning mechanism are proposed.The aim of this paper is to simplify the program complexity of the controller by reducing the number of fuzzy sets of the membership functions(MFs) without losing the system performance and stability via the adjustable controller gain.ZSI exhibits both voltagebuck and voltage-boost capability. It reduces line harmonics, improves reliability, and extends output voltage range.The output $S F$ of the controller can be tuned continuously by a gain updating factor, whose value is derived from fuzzy logic, with the plant error and error change ratio as input variables. Then the expected results, carried out on a four-phase 6/8 pole SRM based on the ASPACEDS1104 platform, to show the feasibility and effectiveness of the devised methods.
\end{abstract}

Keywords: Fuzzy logic controller, scaling factor (SF), switched reluctance motor (SRM), variable-speed drives.

\section{Introduction}

As the development of high speed microcontrollers with powerful computation capability, switched reluctance motor (SRM) drives are under consideration in various applications requiring high performance.SRMs inherently feature numerous merits like simple and rugged structure, being maintenance free, high torque-inertia ratio, fault-tolerance robustness and reliability, high efficiency over a wide range of speeds, etc.The requirements for variable-speed SRM drives include good dynamic and steady-state responses, minimum torque ripple, low-speed oscillation, and robustness. However, due to the heavy nonlinearity of the electromagnetic property and the coupling relationships among flux linkage, torque, and rotor position, it is not easy for an SRM to get satisfactory control characteristics. Therefore, new structure designs [1],high performance magnetic cores [2].

Intelligent control techniques such as fuzzy logic control (FLC), neural network control, or genetic algorithm may allow better performance. Intelligent control approaches try to imitate and learn the experience of the human expert to get satisfactory performance for the controlled plant [3]. One of the most powerful tools that can translate linguistic control rules into practical operation mechanism is the FLC. It has been shown that fuzzy control can reduce hardware and cost and provide better performance than the classical PI, PD, or PID controllers [6]. Recently, fuzzy control theory has been widely studied, and various types of fuzzy controllers have also been proposed for the SRM to improve the drive performance further [3],[7]-[9].Performance of the FLC are scaling factor (SF) tuning, rule base modification, inference mechanism improvement, and membership function redefinition and shifting. Among these techniques, SF tuning is the most used approach, and it has a significant impact on the performance of an FLC. The initial parameters and scaling gains of the controller are optimized by the genetic algorithm to minimize overshoot, settling time, and rising time.

An adaptive fuzzy controller for torque-ripple minimization is presented by Mir et al. [4]. Aiming at torque-ripple minimization, the controller is independent from the accurate SRM model and can adapt to the change of motor characteristics. These characteristics include position error robustness, avoidance of negative torque production, and torque-ripple minimization. This study was aimed at reducing the torque ripple and acoustic noise by an efficient fuzzy control algorithm. An adaptive FLC with scaling gain tuning is proposed in [8]. The universe of discourse (UOD) of the fuzzy sets can be tuned by altering the scaling gain according to the input variables. This significantly improves the system transient and steady-state responses. Koblara proposed a fuzzy logic speed controller for SRM drives [9].Fuzzy controller can produce smooth torque and improve the system performance.Z-source inverter system for adjustable speed drives (ASD)[11]. It can produce any desired output ac voltage, even greater than the line voltage.It reduces line harmonics, and extends output voltage range.It is a relatively recent converter topology that exhibits both voltage-buck and voltage-boost capability[13].ZSI is designed suitable for wind power conversion system[10].The main challenge in wind power system to maintain a constant voltage at the output with unpredictable variation in wind speed,is suitably 
taken care in steady state through buck-boost-capability of (ZSI). The most concerning disturbances affecting the quality of the power in the distribution system are voltage sag/swell [12].The ZSI uses an LC impedance grid, prepares the possibility of voltage buck and boost by short circuiting the inverter legs. Additionally a fuzzy logic control scheme for Z-source inverter based DVR is proposed to obtain desired injecting voltage.

\subsection{SRM Behavior Model}

\section{SRM Drive System}

In SRM, the torque is generated due to the push-pull between reluctance forces. The produced electromagnetic torque is related to the variation of the machine coenergy, and the coenergy varies with the flux linkage, excitation current, and rotor position. The flux linkage, inductance, and torque are highly coupled and nonlinear with the variation of rotor position and phase current, and hence, its magnetization characteristics and operating behavior are difficult to decouple and model mathematically. Fig. 1(a) and (b) shows the crosssectional profile of a four-phase 6/8-pole SRM and the equivalent circuit of one phase winding, respectively.

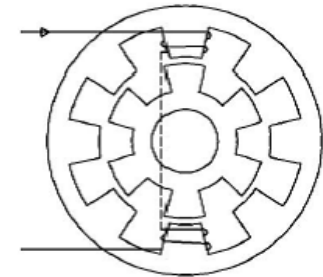

(a)

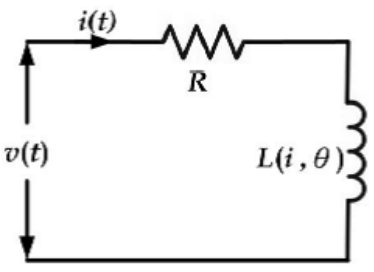

(b)

Fig 1: (a) Cross-sectional profile and (b) equivalent circuit of a 6/8-pole SRM

The equivalent circuit can be represented by a resistance $\mathrm{R}$ in series with an inductance $\mathrm{L}(\mathrm{i}, \theta)$, which is a function of rotor position $\theta$ and excitation current $i$. From Fig. 1(b), the phase voltage can be expressed by

With

$$
\mathrm{V}(\mathrm{t})=\mathrm{R} \cdot \mathrm{i}(\mathrm{t})+\frac{d \lambda(i, \theta)}{d t}
$$

$$
\lambda(\mathrm{i}, \theta)=\mathrm{L}(\mathrm{i}, \theta) \mathrm{i}
$$

Where $\lambda$ is the flux linkage, which is dependent on $i$ and $\theta . v(t), i(t)$, and $L(i, \theta)$ are the instantaneous voltage across the excited phase winding, the excitation current, and the self-inductance, respectively. According to (1) and (2), the dynamic behavior of the $m$-phase SRM can be denoted as

$$
\mathrm{Vk}=\sum_{j=1}^{m}\left\{\left(\mathrm{Rk}+\omega \frac{\partial \mathrm{Lkj}}{\partial \theta}\right) \mathrm{ij}+\left(\mathrm{Lkj}+\mathrm{ij} \frac{\partial \mathrm{Lkj}}{\partial \mathrm{ij}}\right) \frac{\partial \mathrm{ij}}{\partial t} \quad \mathrm{k}=1,2, \ldots \ldots \mathrm{m}\right.
$$

where $\omega$ is the rotor angular velocity and $m$ is the phase number.

The obtained coenergy is equal to the area enclosed by the $\lambda-i$ curve over one excitation cycle and can be calculated by

$$
\text { Wce }(\mathrm{i}, \theta)=\int_{0}^{i} \lambda(i, \theta) d i \mid \theta=\text { constant }
$$

For a specified current, the induced electromagnetic torque can be obtained by differentiating the coenergy Wce with respect to the rotor position $\theta$, which can be expressed as

$$
\operatorname{Te}(\mathrm{i}, \theta)=\frac{\left.\partial \text { Wce }^{(} \mathrm{i}, \theta\right)}{\partial \theta} \mid \mathrm{i}=\text { constant }
$$

Here, we define an incomplete torque function as

$$
\mathrm{Tk}=\frac{1}{2} \sum_{j=1}^{m}\left\{\operatorname{sgn}(\mathrm{k}, \mathrm{j}) \mathrm{ij} \frac{\partial \mathrm{Lkj}}{\partial \theta}\right\}
$$

$$
\mathrm{k}=1,2, \ldots \ldots \ldots \mathrm{m}
$$


Where

$$
\operatorname{sgn}(k, j)=\left\{\begin{array}{lll}
1, & \text { if } & k=j \\
-1, & \text { if } \quad k \neq j
\end{array}\right.
$$

From (2) and (4)-(7), including the mutual inductance, the produced totally electromagnetic torque can be denoted as

$$
\mathrm{Te}=\sum_{k=1}^{m} \quad \mathrm{i}_{\mathrm{k}} \mathrm{T}_{\mathrm{k}}
$$

The mechanical torque of the rotor can be expressed as

$$
\mathrm{Tmec}=\mathrm{Te}-\mathrm{B} \omega-\mathrm{J} \frac{d \omega}{d t}
$$

Where $J, B$, and Tmec stand for the machine's moment inertia, friction coefficient, and mechanical torque, respectively. Together with (3), (6), (8), and (9), the matrix-form behavior model of the SRM, taking the magnetic coupling effect into account, can be denoted by (10), If the mutual inductance is negligible, then

$$
\begin{aligned}
& \left\{\begin{array}{c}
L k j=0, \quad \text { if } \quad k \neq j \\
L k j \neq 0, \quad \text { if } \quad k=j
\end{array}\right. \\
& {\left[\begin{array}{c}
v_{1} \\
v_{2} \\
\vdots \\
v_{m} \\
T_{\mathrm{mec}}
\end{array}\right]=\left[\begin{array}{ccccc}
\left(R_{1}+\omega \frac{\partial L_{11}}{\partial \theta}\right) & \omega \frac{\partial L_{12}}{\partial \theta} & \cdots & \omega \frac{\partial L_{1 m}}{\partial \theta} & 0 \\
\omega \frac{\partial L_{21}}{\partial \theta} & \left(R_{2}+\omega \frac{\partial L_{22}}{\partial \theta}\right) & \cdots & \omega \frac{\partial L_{2 m}}{\partial \theta} & 0 \\
\vdots & \vdots & & \vdots & \vdots \\
\omega \frac{\partial L_{m 1}}{\partial \theta} & \omega \frac{\partial L_{m 2}}{\partial \theta} & \cdots & \omega \frac{\partial L_{m m}}{\partial \theta} & 0 \\
T_{1} & T_{2} & \cdots & T_{m} & -B
\end{array}\right]\left[\begin{array}{c}
i_{1} \\
i_{2} \\
\vdots \\
i_{m} \\
\omega
\end{array}\right]} \\
& +\left[\begin{array}{ccccc}
\left(L_{11}+i_{1} \frac{\partial L_{11}}{\partial i_{1}}\right) & \left(L_{12}+i_{2} \frac{\partial L_{12}}{\partial i_{2}}\right) & \ldots & \left(L_{1 m}+i_{m} \frac{\partial L_{1 m}}{\partial i_{m}}\right) & 0 \\
\left(L_{21}+i_{1} \frac{\partial L_{21}}{\partial i_{1}}\right) & \left(L_{22}+i_{2} \frac{\partial L_{22}}{\partial i_{2}}\right) & \ldots & \left(L_{2 m}+i_{m} \frac{\partial L_{2 m}}{\partial i_{m}}\right) & 0 \\
\vdots & \vdots & \vdots & \vdots \\
\left(L_{m 1}+i_{1} \frac{\partial L_{m 1}}{\partial i_{1}}\right) & \left(L_{m 2}+i_{2} \frac{\partial L_{m 2}}{\partial i_{2}}\right) & \ldots & \left(L_{m m}+i_{m} \frac{\partial L_{m m}}{\partial i_{m}}\right) & 0 \\
0 & 0 & \ldots & 0 & -J
\end{array}\right] \frac{d}{d t}\left[\begin{array}{c}
i_{1} \\
i_{2} \\
\vdots \\
i_{m} \\
\omega
\end{array}\right]
\end{aligned}
$$

\subsection{Drive System Architecture}

Shown in Fig. 2 is the configuration of the studied SRM drive system. It consists of four controllers, which include the fuzzy speed controller, the PI current controller, the exciting angle regulation controller, and the commutation logic controller; a gate driver circuit with photo couplers; a power inverter; and the four-phase 6/8-pole SRM. The fuzzy speed controller receives the speed error signal and converts it into four-phase current commands that will be sent to the current controller. The actual current, sensed by the Hall-effect sensor, is compared with the current command to obtain the current error. According to the error value, the pulse width modulation gating signals of insulated-gate bipolar transistors in an asymmetric half-bridge power inverter are generated by the current controller. The gating signals drive the power inverter through the photo coupler isolation. Z-source inverter system for adjustable speed drives(ASD)it can produce any desired output ac voltage,even greater than the line voltage.It reduces line harmonics,and extends output voltage range.ZSI exhibits both voltage-buck and voltage-boost capability. With the inputs of actual speed, speed errors, current, and rotor position, both algorithms of torque iterative learning control (TILC) and energy iterative learning control (EILC) are run to minimize the torque ripple and energy conversion loss by regulating the incremental turn-on and turn-off angles ( $\Delta \theta$ on, $\Delta \theta$ off) and the duty cycle $(D)$ to enhance the driving performance. When the speed error (werror) is acceptably low, the whole exciting angle regulation controller will be enabled to run the TILC and EILC processes and to compensate the parameter variations caused by the inaccurate motor model. The commutation logic controller is used to derive and determine the phase commutation moment according to the rotor position, excitation turn-on angle, and turn-off angle. In order to simplify the hardware complexity, all of the four controllers are implemented on a DSP-based dSPACE control platform. 


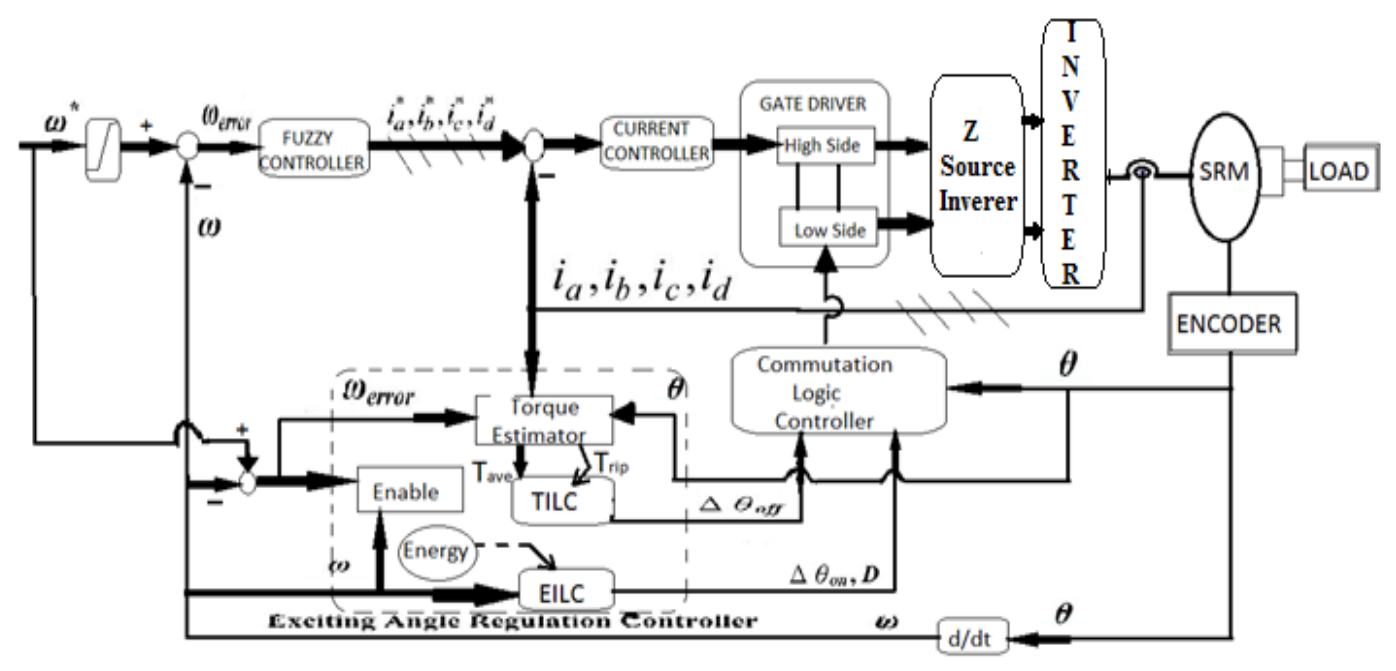

Fig 2: Architecture of the SRM drive system.

\section{Fuzzy Logic Controller Design}

In this section, the fuzzy control fundamentals will be outlined first, and then, the key point of selftuning PI-like fuzzy controller (STFC) will be briefly reviewed. Afterward, the modified design of the proposed STFC will be described in detail.

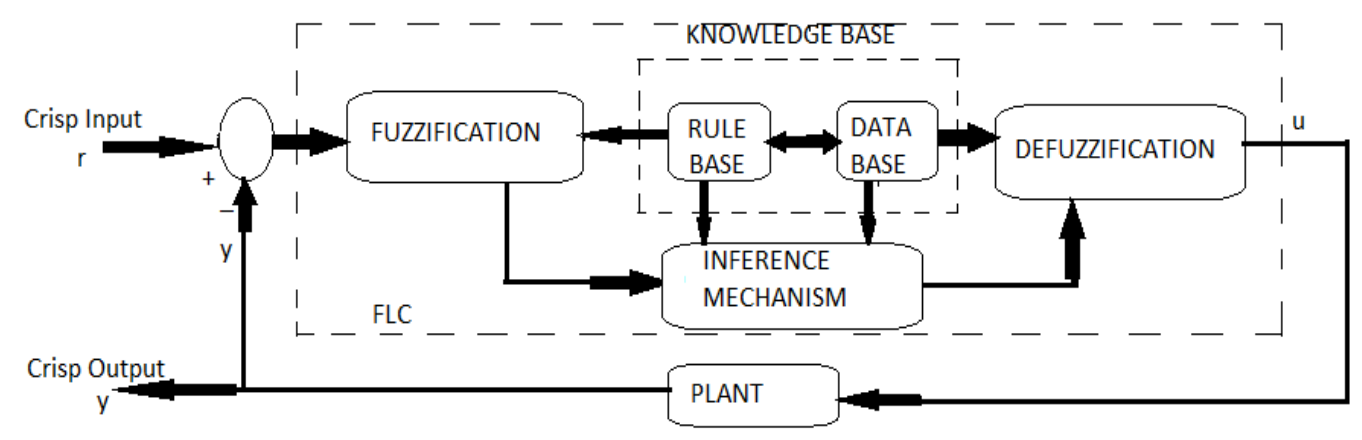

Fig 3: Basic structure of a fuzzy logic control system

\subsection{Fuzzy Control Philosophy}

A basic FLC system structure, which consists of the knowledge base, the inference mechanism, the fuzzification interface, and the defuzzification interface, is shown in Fig. 3. Essentially, the fuzzy controller can be viewed as an artificial decision maker that operates in a closed-loop system in real time. It grabs plant output $y(t)$, compares it to the desired input $r(t)$, and then decides what the plant input (or controller output) $u(t)$ should be to assure the requested performance. The inputs and outputs are "crisp." The fuzzification block converts the crisp inputs to fuzzy sets, and the defuzzification block returns these fuzzy conclusions back into the crisp outputs. Inference engine using if-then type fuzzy rules converts the fuzzy input to the fuzzy output.

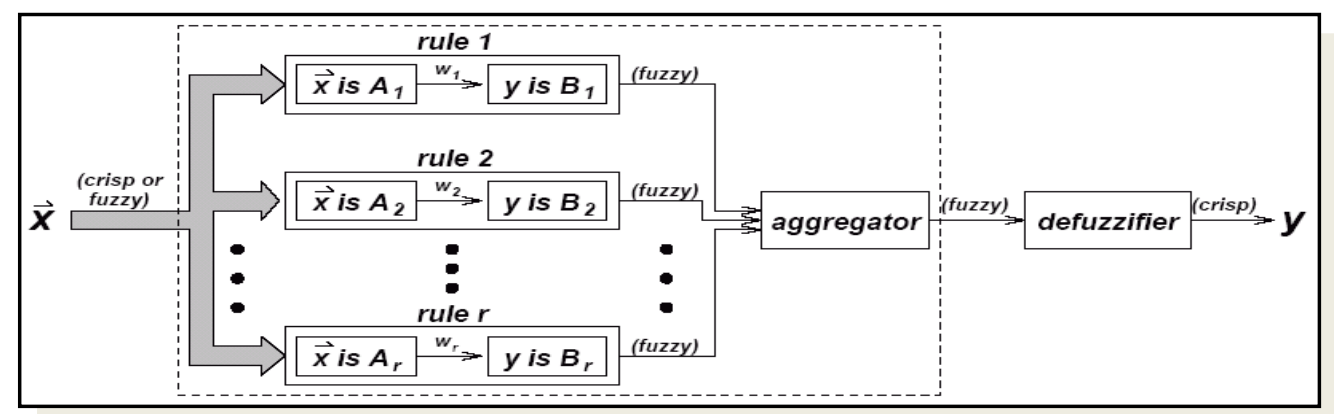

Fig 4: Basic structure of an Inference engine 


\section{2 .Overview of Self-Tuning FLC}

The PI-like fuzzy controller (PIFC) is driven by a set of control rules rather than constant proportional and integral gains. The block diagrams of a conventional PIFC and an STFC are shown in Figs. 5 and 6, respectively. The main difference between both controllers is that the STFC includes another control rule base for the gain updating factor $\alpha$ [14]. Adaptability is necessary for fuzzy controllers to ensure acceptable control performance over a wide range of load variations regardless of inaccurate operating knowledge or plant dynamic behaviour. These are the commonly used methods to make a fuzzy controller adaptive: input or output SF tuning, MF definition and control rule modification. In a classical fuzzy controller, the UOD tuning of the MFs of the input or output variables can be used to overcome the steady-state error.Here, a discrete-time controller with two inputs and a single output is considered. From Fig 6, the error $e$ and change of error $\Delta e$ are used as the input variables, which are defined as,

$$
\begin{aligned}
\mathrm{e}(\mathrm{k}) & =\mathrm{r}(\mathrm{k})-\mathrm{y}(\mathrm{k}) \\
\Delta \mathrm{e}(\mathrm{k}) & =\mathrm{e}(\mathrm{k})-\mathrm{e}(\mathrm{k}-1) \\
& =\mathrm{y}(\mathrm{k}-1)-\mathrm{y}(\mathrm{k})
\end{aligned}
$$

where $\mathrm{r}$ and $\mathrm{y}$ denote the reference command and plant output, respectively. Indices $\mathrm{k}$ and $\mathrm{k}-1$ represent the current and previous states of the system, respectively. The controller output is the incremental change of the control signal $\Delta \mathrm{u}(\mathrm{k})$. The control signal can be obtained by

$\mathrm{u}(\mathrm{k})=\mathrm{u}(\mathrm{k}-1)+\Delta \mathrm{u}(\mathrm{k})$

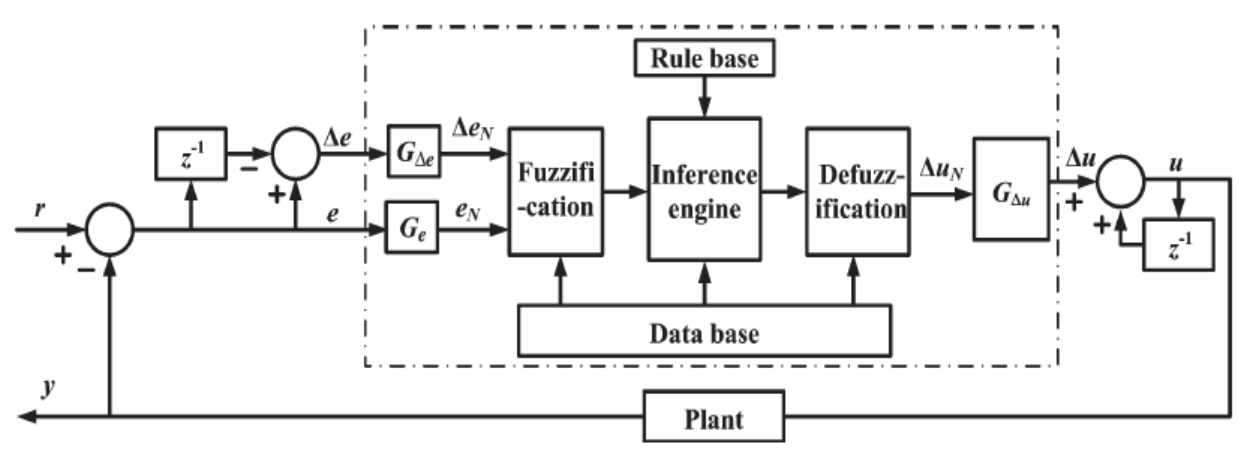

Fig 5: Block diagram of a conventional PIFC.

The UOD in all membership functions of the controller inputs, i.e., $e$ and $\Delta e$, and output, i.e., $\Delta u$, are defined on the normalised domain with the gain updating factor $\alpha$ (which is utilized to fine tune the output SF) over the interval $[0,1]$, as shown in Fig. 7 . The linguistic values NB, NS, ZE, PS, and PB stand for negative big, negative small, zero, positive small, and positive big, respectively. Here, symmetric triangles with equal bases and $50 \%$ overlap with adjacent MFs are chosen. The SFs $G e, G \Delta e$, and $G \Delta u$, which perform the specific normalization of input and output variables, play a role equivalent to that of the gains of a conventional controller. Hence, they hold the dominant impact on controller stability and performance.

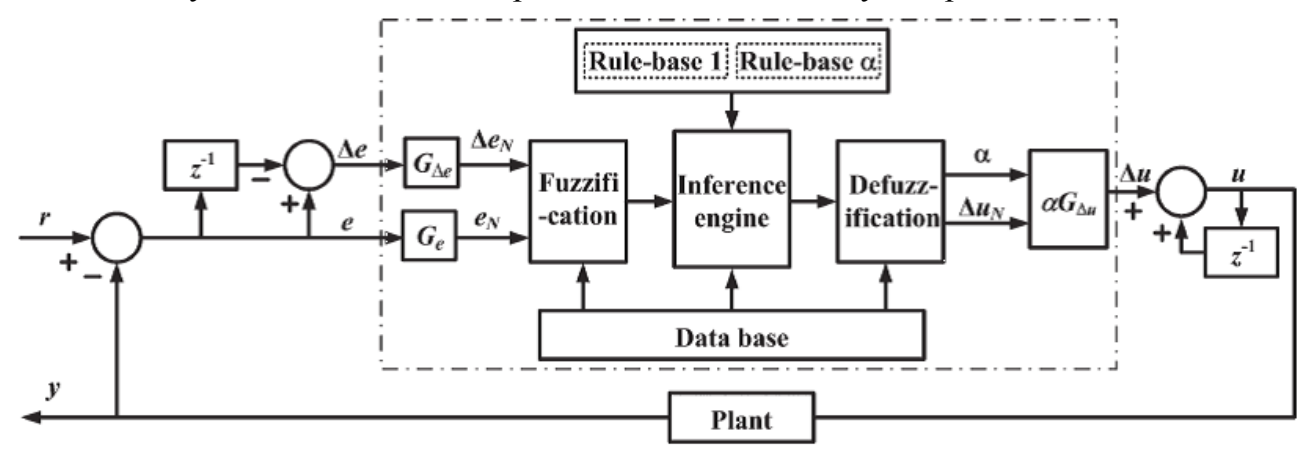

Fig 6: Block diagram of an STFC 
TABLE 1: Rule Base (7x7) for Deriving Output Variable $\boldsymbol{\Delta} \mathbf{U}$

\begin{tabular}{|l|l|l|l|l|l|l|l|}
\hline \multicolumn{1}{|r|}{$e^{\alpha}$} & NB & NM & NS & ZE & PS & PM & PB \\
\hline NB & NB & NB & NB & NM & NS & NS & ZE \\
\hline NM & NB & NM & NM & NM & NS & ZE & PS \\
\hline NS & NB & NM & NS & NS & ZE & PS & PM \\
\hline ZE & NB & NM & NS & ZE & PS & PM & PB \\
\hline PS & NM & NS & ZE & PS & PS & PM & PB \\
\hline PM & NS & ZE & PS & PM & PM & PM & PB \\
\hline PB & ZE & PS & PS & PM & PB & PB & PB \\
\hline
\end{tabular}

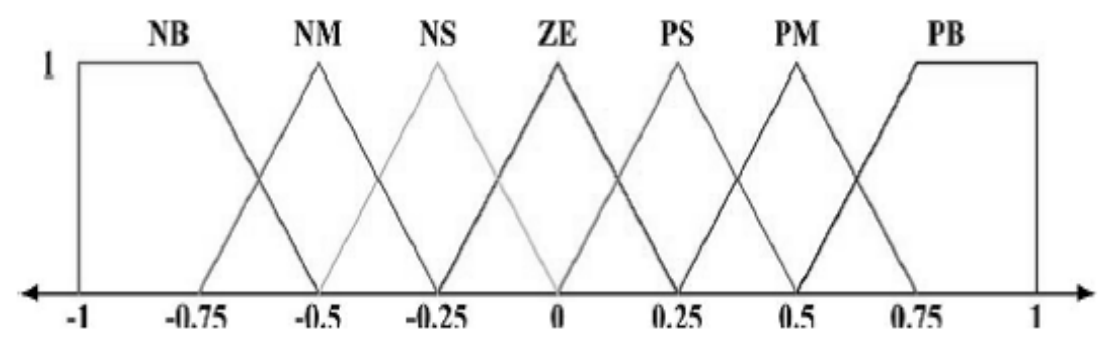

Fig 7: Membership functions of $e, \Delta e$, and $\Delta u$ with gain updating factor $\alpha$ of $7 \times 7$ rule base

TABLE 2: Rule Base (5x5) for Deriving Output Variable $\mathbf{\Delta} \mathbf{U}$

\begin{tabular}{|c|c|c|c|c|c|c|}
\hline & $\Delta e$ & \multirow[t]{2}{*}{ NB } & \multirow[t]{2}{*}{ NS } & \multirow[t]{2}{*}{$\overline{Z E}$} & \multirow[t]{2}{*}{ PS } & \multirow[t]{2}{*}{$\mathrm{PB}$} \\
\hline$e$ & $\mathrm{o} / \mathrm{p}$ & & & & & \\
\hline $\mathrm{NE}$ & & NB & NB & NS & NS & ZE \\
\hline $\mathrm{NS}$ & & $\mathrm{NB}$ & NS & NS & ZE & PS \\
\hline$\overline{\mathrm{ZE}}$ & & $\mathrm{NS}$ & $\mathrm{NS}$ & $\mathrm{ZE}$ & PS & PS \\
\hline $\mathrm{PS}$ & & NS & ZE & PS & PS & PB \\
\hline$\overline{\mathrm{PB}}$ & & ZE & PS & PS & $\mathrm{PB}$ & $\mathrm{PB}$ \\
\hline
\end{tabular}

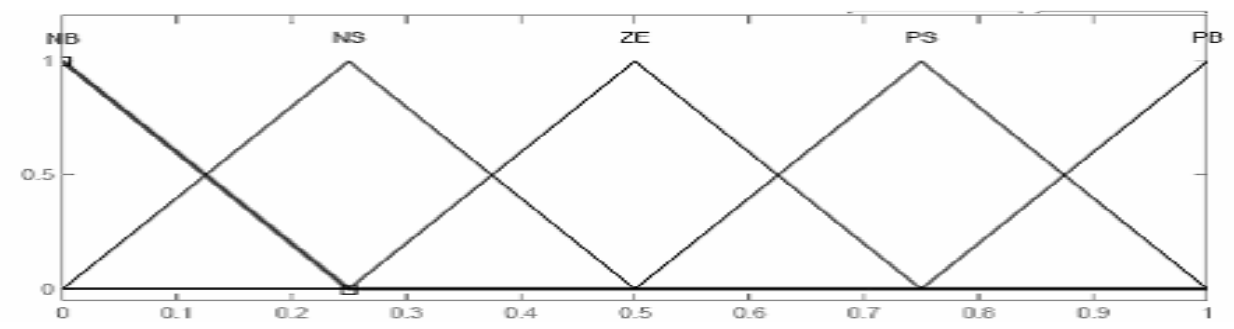

Fig 8: Membership functions of $e, \Delta e$, and $\Delta u$ with gain updating factor $\alpha$ of $5 \times 5$ rule base 
The MFs for both normalized inputs $(e N$ and $\Delta e N)$ and output $(\Delta u N)$ of the controller have been defined on the normalized domain $[-1,1]$.For conventional FLCs, the controller output $(\Delta u N)$ is mapped onto the respective actual output $(\Delta u)$ domain by the output SF $G \Delta u$. On the other hand, the actual output of the self tuning FLC is obtained by using the effective SF $\alpha G \Delta u$ [14]. Hence, adjusting the SFs can modify the corresponding UODs of the control variables. The adequate values of the input and output SFs can be derived based on the professional experience from the plant under control. It can also be derived through trial and error to achieve the best acceptable control performance. As shown in Fig.6, the relationships between the SFs and the input and output variables of the STFC can be expressed as follows:

$$
\begin{aligned}
\mathrm{e}_{\mathrm{N}} & =\mathrm{Gee} \\
\Delta \mathrm{e}_{\mathrm{N}} & =\mathrm{G} \Delta \mathrm{e} \Delta \mathrm{e} \\
\Delta \mathrm{u} & =(\alpha \mathrm{G} \Delta \mathrm{u}) \Delta \mathrm{u}_{\mathrm{N}}
\end{aligned}
$$

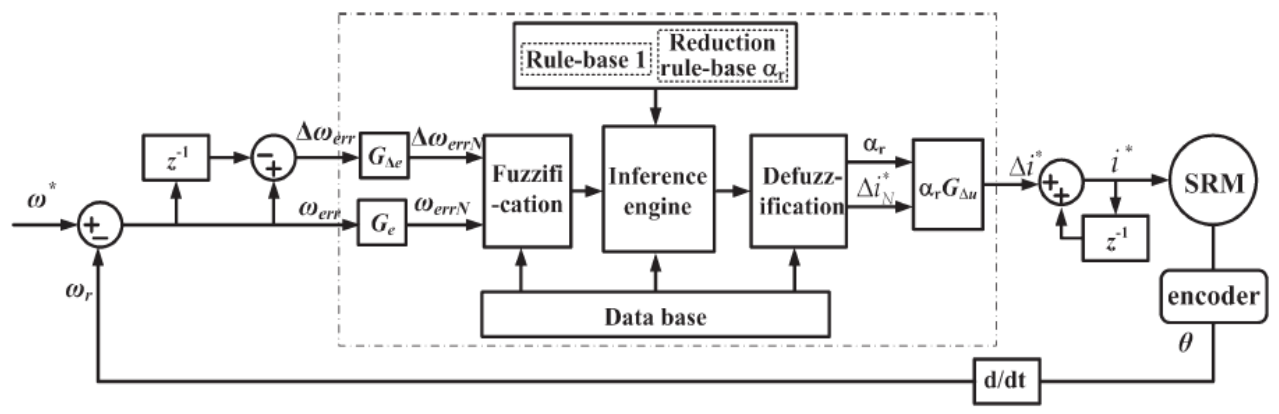

Fig 9: Block diagram of the proposed FLC with a reduction of the control rule

\subsection{Self-Tuning FLC with Control Rule Reduction}

This work presents a simple but robust model-independent self-tuning mechanism for FLCs with the most important feature that it depends neither on the process being controlled nor on the controller used. The control algorithm must be implemented on the microcontroller with limited memory space and computational capability. The rule base of the STFC proposed in[14] choose five fuzzy sets for each membership function of the input variables $e$ and $\Delta e$. Twenty-five fuzzy rules are needed for deriving controller output $\Delta u$ and $\alpha$, respectively. This is a challenge to the performances of the used DSP or micro-controller unit. This paper focuses on, first, the reduction of the number of fuzzy rules for deriving $\alpha$ and, second, the simplification of the memory requirement and computational complexity of the designed controller. The architecture of the proposed controller with control rule reduction is shown in Fig. 9.

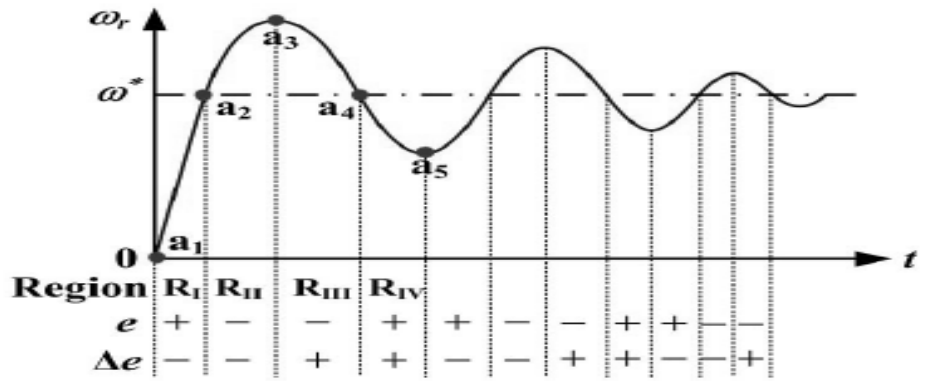

Fig 10: Dynamic behavior of motor step response of $7 \times 7$ rule base

The dynamic behavior of motor step response of $7 \times 7$ rule base as shown in fig 10 . The speed response can be roughly divided into four regions, i.e., RI-RIV, and two sets of particular points, i.e., crossover points (a2, a4) and peak points (a3, a5). According to the definitions of (12) and (13), the signs of $e$ and $\Delta e$ will change when the response curve passes through the different regions. For example, the sign of $(e, \Delta e)$ in regions RII and RIV are $(-,-)$ and $(+,+)$, respectively. The states in these two regions mean that the speed now is not only upward (RII) or downward (RIV) far away from the speed command but are also going farther away from it. In this situation, the controller should provide large gain $(\alpha \mathrm{r} G \Delta u)$ to prevent worsening the condition. Therefore the dynamic behaviour of motor step response will attain its steady state very slowly by using $(7 \times 7=49)$ rules. This 
may result in long settling time and oscillation around the preset speed when the system approaches the steady state.

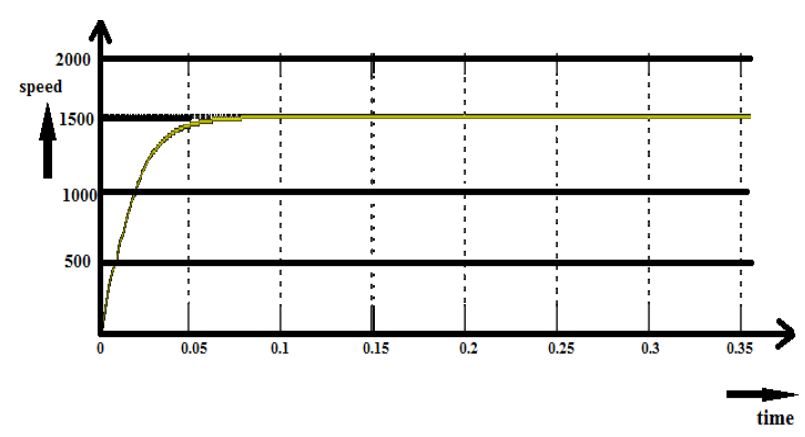

Fig 11: Dynamic behavior of motor step response of Control Rule Reduction

But here, the focus is on the reduction of rule numbers for deriving updating factor $\alpha \mathrm{r}$. In the proposed scheme, to reduce the rule numbers, a practical observation of the motor step response, as shown in Fig 11. The proposed frame of the reduced rule base for deriving $\alpha \mathrm{r}$ is shown in Table 1. In this proposed scheme, although there are 25 control rules, in practical cases when the control process is running, according to the speed command, the program will be switched to run a subroutine, which is instructed to correspond to one of the five speed command ranges. Each time, only five control rules are used to derive $\alpha \mathrm{r}$ when the chosen subroutine is executed. Therefore the dynamic behaviour of motor step response of control rule reduction will attain its steady state very quickly by using $(5 \times 5=25)$ rules. This may result in short settling time and there is very small oscillation around the preset speed when the system approaches the steady state. Consequently, when the control procedure is running, the numbers of control rules used to derive $\Delta i^{*}$. The Mamdani-type inferential method cooperating with the center-of-area defuzzification procedure is utilized to produce the crisp controller output.

\subsection{Gain Tuning Strategy}

The PIFC without scaling gain tuning mechanism has a drawback, i.e., as the controller design is finished, the defined domain of the input and output variables is fixed. This may result in long settling time and oscillation around the preset speed when the system approaches the steady state. This is caused by low controller resolution when the speed error is small. In order to obtain satisfactory performance, the UOD of the controller should be adjusted according to the operating point. Therefore, the fuzzy controller, which can change UOD by tuning scaling gains through a continuous and nonlinear variation of the updating factor, is developed. Here, the attention is focused on the tuning of output scaling gain because it is equivalent to the controller gain, and output-gain regulation plays a dominant role due to its strong impact on the overall performance of the controller. The self-adjusting mechanism of the proposed fuzzy controllers is described as follows:

3.4.1. Variation Effect of Input and Output SFs: SF modulation is one of the most employed solutions to enhance the performance of a fuzzy controller. The design of the SFs, particularly the output SF, is very crucial in an FLC because of their influences on the performance. Take the STFC shown in Fig 6 as an example. Converting $e, \Delta e$, and $\Delta u$ into $e N, \Delta e N$, and $\Delta u N$ by the scaling gains, respectively, means that they are transferred from the actual UOD into the interval [0,1] (normalization). The effect of SF adjustment is equivalent to extending or shrinking the actual UOD of the input and output variables.

3.4.2. Self-Tuning Mechanism: The systemic methods for gain tuning to obtain the optimal response because the determination of the optimal values of the adjustable parameters requires the knowledge of a precise model of the plant. Moreover, the practical testing results show that the effect of input SF tuning has lesser impact on system performance than output SF tuning, and both types of SF tuning will increase the system complexity further. Therefore, only output SF tuning is adopted in the proposed two modified STFCs. The design guidelines are described as follows:

Step 1: For the proposed two FLCs, the proper initial values of $G e, G \Delta e$, and $G \Delta u$ can be obtained by (18) for control rule reduction Choosing $G e G \Delta e$ and $G \Delta u$ to cover the whole normalized domain $[-1,1]$ or interval [emin, emax]. An appropriate initial operating condition is obtained when a good transient response is achieved.

$$
\begin{aligned}
\mathrm{Ge} & =\frac{1}{\omega e r r, \max }\left(\text { or } \frac{1}{|\omega e r r, \min |}\right) \\
\mathrm{G} \Delta \mathrm{e} & =\frac{1}{\Delta \omega \operatorname{err}_{, \max }} \mathrm{G} \Delta \mathrm{u}=\Delta \mathrm{umax}
\end{aligned}
$$


Step 2: Keep the values of $G e, G \Delta e$, and $G \Delta u$ the same as those in Step 1), and begin to tune the updating factor, where $\alpha$ index is $\alpha \mathrm{r}$ or $\alpha$ i represents the updating factor for the FLC with control rule reduction. $f \alpha$ index is a nonlinear function defined on the $e$ and $\Delta e$ planes, and $k \Delta u$ is the scaling constant of $G \Delta u$. Here, $G \Delta u$ is set $k \Delta u$ times greater than that obtained in Step 1). The determination of $k \Delta u$ is empirical. At the same time, the output SF can be fine-tuned by altering the value of $\alpha$ index to achieve a relatively small but satisfactory output. On the other hand, if we want to shorten the rise time, the $k \Delta u$ in (19) should be set larger. A large value of $k \Delta u G \Delta u$ is utilized in the proposed schemes to counteract the effect that $\alpha$ always lies in $[0,1]$ and guarantee a faster response with relatively small overshoot.

$$
\begin{aligned}
\Delta \mathrm{u}(\mathrm{k}) & =\operatorname{\alpha index}(\mathrm{k})(\mathrm{k} \Delta \mathrm{uG} \Delta \mathrm{u}) \Delta \mathrm{uN} \\
\alpha \operatorname{index}(\mathrm{k}) & =\text { faindex }(\mathrm{e}(\mathrm{k}), \Delta \mathrm{e}(\mathrm{k}))
\end{aligned}
$$

Finally, the performance will be compared to shows that the STFC with control rule reduction is the best among the existing methods and also demonstrates that the proposed controller can quickly regulate itself to adapt to the current environmental change.

\section{Conclusion}

In this paper, based on the improved performance of the closed loop control of Switched Reluctance Motor drives using Z-Source Inverter with the control rule reduction of 5x5 using Fuzzy Logic Controller(FLC) with the output scaling factor (SF) self-tuning mechanism by altering a gain updating factor has been devised. The modified rule bases are designed to simplify the program complexity of the controller by reducing the number of fuzzy sets of the membership functions without losing the system performance and stability using the adjustable controller gain. Z-source inverter, that exhibits both voltage-buck and voltage-boost capability.It reduces line harmonics, improves reliability, and extends output voltage range. The rule bases are based on the fuzzy control rules, which are derived from the practical understanding of the SRM's basic behaviour, operating experience. Here, attention is focused on the adjustment of the output SF because output-gain regulation has higher impact on the performance and stability of the system and the proposed controller can also simplify the complexity of the control system.Based on the dSPACE DS1104 platform, tests on a four-phase 6/8- pole SRM under the speed set-point change and load disturbance have been carried out to measure various performance indices such as peak overshoot or undershoot, steady-state error, rise time, settling time, etc. The expected results of the proposed control, shows very good stability and robustness against speed and load variations over a wide range of operating conditions and also the performance of the proposed controllers will be compared with conventional counterpart.

\section{References}

[1] P. C. Desai, M. Krishnamurthy, N. Schofield, and A. Emadi, "Novel switched reluctance machine configuration with higher number of rotor poles than stator poles: Concept to implementation,” IEEE Trans. Ind. Electron., vol. 57, no. 2, pp. 649-659, Feb. 2010 .

[2] J. Corda and S. M. Jamil, "Experimental determination of equivalent circuit parameters of a tubular switched reluctance machine with solid-steel magnetic core," IEEE Trans. Ind. Electron., vol. 57, no. 1, pp. 304-310, Jan. 2010.

[3] P. Vas, Artificial-Intelligence-Based Electrical Machines and Drives: Application of Fuzzy, Neural, Fuzzy-Neural, and GeneticAlgorithm-Based Techniques. London, U.K.: Oxford Univ. Press, 1999.

[4] S. Mir, M. E. Elbuluk, and I. Husain, "Torque-ripple minimization in switched reluctance motors using adaptive fuzzy control," IEEE Trans. Ind. Appl., vol. 35, no. 2, pp. 461-468, Mar./Apr. 1999.

[5] R. K. Mudi and N. R. Pal, “A robust self-tuning scheme for PI- and PD type fuzzy controllers, ” IEEE Trans. Fuzzy Syst., vol. 7, no. 1, pp. 2-16, Feb. 1999

[6] A. G. Perry, G. Feng, Y. F. Liu, and P. C. Sen, “A design method for PI-like fuzzy logic controller for DC-DC converter,” IEEE Trans. Ind. Electron., vol. 54, no. 1, pp. 190-199, Feb. 2007.

[7] S. Chowdhuri, S. K. Biswas, and A. Mukherjee, "Performance studies of fuzzy logic based PI-like controller designed for speed control of switched reluctance motor," in Proc. IEEE Int. Conf. Ind. Electron. Appl., 2006, pp. 1-5.

[8] J. Xiu and C. Xia, "An application of adaptive fuzzy logic controller for switched reluctance motor drive," in Proc. IEEE Fuzzy Syst. Knowl. Disc., 2007, pp. 154-158.

[9] T. Koblara, “Implementation of speed controller for switched reluctance motor drive using fuzzy logic," in Proc. OPTIM, 2008, pp. $101-105$.

[10] Santosh sonar and Tanmoy maity, “Z-source Inverter based control of Wind Power, ” presented at the IEEE Industry Applications Soc. Annu. Meeting,2011.

[11] Fang Zheng Peng,Alan Joseph, JinWang, Miaosen Shen, Lihua Chen, Zhiguo Pan, Eduardo Ortiz-Rivera, and Yi Huang, “Z-Source Inverter for Motor Drives," IEEE Trans. Power Electron., vol.20, no. 4,pp.857-863, July 2005.

[12] M.Balamurugan, T.S. Sivakumaran, and M.Aishwariya Devi, "Voltage Sag/Swell Compensation Using Z-source Inverter DVR based on FUZZY Controller," presented at IEEE International Conference on Emerging Trends in Computing, Communication and Nanotechnology, pp.648-653,2013.

[13] Francis Boafo Effah, Patrick Wheeler, Jon Clare, and Alan Watson, "Space-Vector-Modulated Three-Level Inverters With a Single Z-Source Network,” IEEE Trans. PowerElectron.,vol.28, no.6,pp.2806-2815,June2013. 